\title{
Post-traumatic osteolysis of the distal clavicle
}

\author{
Hee Kyung Kim • Eric Crotty
}

Received: 27 May 2009/Revised: 4 August 2009 / Accepted: 24 August 2009 /Published online: 24 September 2009

(C) Springer-Verlag 2009

A 16-year-old boy with shoulder pain after trauma underwent shoulder radiographs, which were considered normal. Pain persisted with conservative treatment and follow-up. Radiograph at 3 months demonstrated periarticular bony erosion of the distal clavicle (arrow) (Fig. 1). Subsequent MRI for evaluation of the acromioclavicular joint demonstrated bone marrow and soft-tissue edema, periarticular erosion of the distal clavicle, adjacent softtissue edema and widening of the joint capsule (arrow) (Fig. 2). Based on the clinical history of trauma and imaging findings, post-traumatic osteolysis of the distal clavicle was diagnosed and confirmed during surgical resection of the distal clavicle. Post-traumatic osteolysis of the distal clavicle results from acute trauma or repetitive microtrauma, and incidence is $6 \%$ in adults with prior

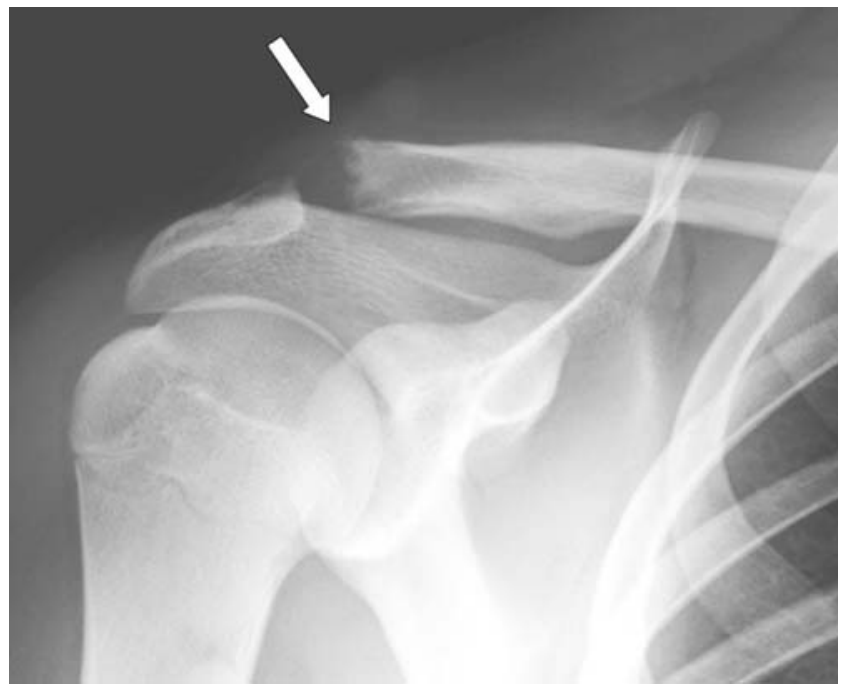

Fig. 1 Follow-up radiograph 3 months after initial trauma

H. K. Kim $(\bowtie) \cdot$ E. Crotty

Department of Radiology, Cincinnati Children's Medical Center,

3333 Burnet Ave.,

Cincinnati, OH 45229, USA

e-mail: hee.kim@cchmc.org acromioclavicular joint separation. It is characterized by progressive bony resorption and bone marrow and softtissue edema of the distal clavicle, although exact pathophysiology is unknown [1]. Usually, good response is achieved by conservative management; surgical resection is performed in refractory cases [2].

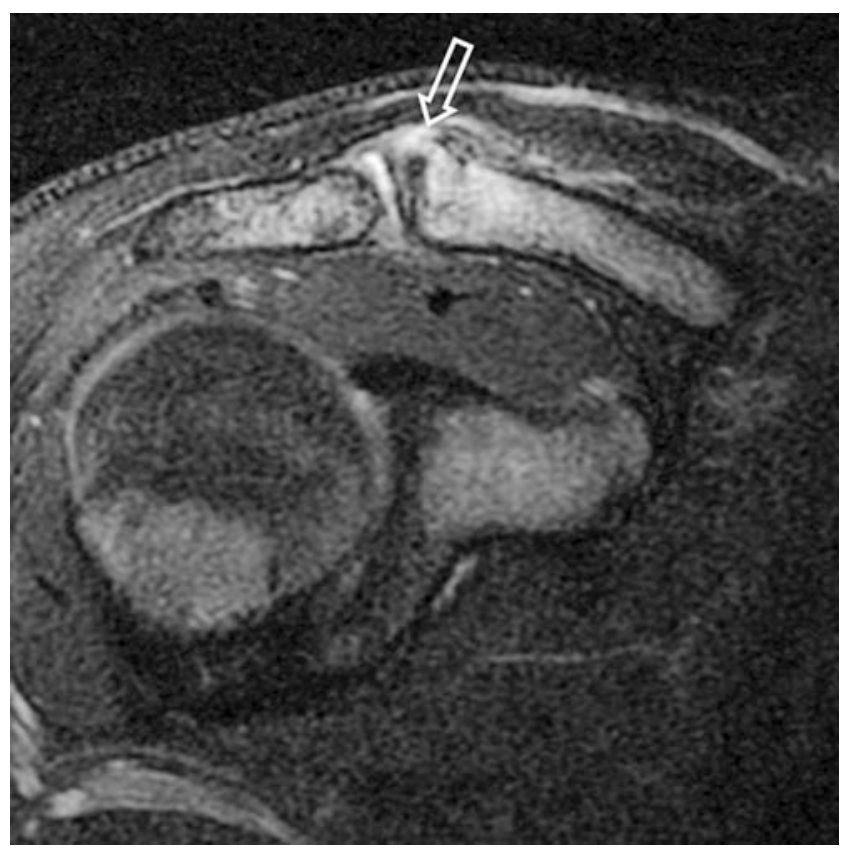

Fig. 2 Oblique coronal T2-W image with fat-suppression of right clavicle

\section{References}

1. Yu YS, Dardani M, Fischer RA (2000) MR observations of postraumatic osteolysis of the distal clavicle after traumatic separation of the acromioclavicular joint. J Comput Assist Tomogr 24:159-164

2. Mestan MA, Bassano JM (2001) Posttraumatic osteolysis of the distal clavicle: analysis of 7 cases and a review of the literature. J Manipulative Physiol Ther 24:356-361 